\title{
Fulminant hepatorenal syndrome due to Acetaminophen toxicity: A
} case report

Zahra Nekoukar ${ }^{1}$, Minoo Moghimi $^{1}$, Zakaria zakariaei ${ }^{1}$, Mahdi Fakhar ${ }^{1}$, and Rabeeh Tabaripour ${ }^{1}$

${ }^{1}$ Mazandaran University of Medical Sciences

January 25, 2021

\begin{abstract}
Hepatorenal syndrome is a rare life-threatening complication of acetaminophen toxicity. It is not responsive to fluid therapy and need performing an emergent liver transplantation. Here, we introduce a 24-year-old woman with a history of chronic high doses of acetaminophen consumption, presenting with nausea, vomiting, lethargy, oliguria, and severe metabolic acidosis.
\end{abstract}

\section{Hosted file}

Acetaminophen toxicity.pdf available at https://authorea.com/users/391700/articles/505734fulminant-hepatorenal-syndrome-due-to-acetaminophen-toxicity-a-case-report 